\title{
The Three Reflections Theorem Revisited
}

\section{The Three Reflections Theorem Revisited ABSTRACT}

It is well-known that, in a Euclidean plane, the product of three reflections is again a reflection, iff their axes pass through a common point. For this "Three reflections Theorem" (3RT) also non-Euclidean versions exist, see e.g. [4]. This article presents affine versions of it, considering a triplet of skew reflections with axes through a common point. It turns out that the essence of all those cases of $3 R T$ is that the three pairs (axis, reflection direction) of the given (skew) reflections can be observed as an involutoric projectivity. For the Euclidean case and its non-Euclidean counterparts this property is automatically fulfilled.

From the projective geometry point of view a (skew) reflection is nothing but a harmonic homology. In the affine situation a reflection is an indirect involutoric transformation, while "direct" or "indirect" makes no sense in projective planes. A harmonic homology allows an interpretation both, as an axial reflection and as a point reflection. Nevertheless, one might study products of three harmonic homologies, which result in a harmonic homology again. Some special mutual positions of axes and centres of the given homologies lead to elations or even to the identity, too.

A consequence of the presented results are further generalisations of the $3 R T$, e.g. in planes with Minkowski metric, affine or projective 3-space, or in circle geometries.

Key words: three reflections theorem, axial reflection, harmonic homology, involutoric projectivity

MSC2010: 51Mxx

\section{Introduction}

According to F. Bachmann [2] reflections can be observed as the basic transformations of a geometry, and the set of these transformations defines at least a sub-geometry of "classical" geometries. Thereby a general transforma-

\section{Nov pogled na teorem tri simetrije SAŽETAK}

$\mathrm{U}$ euklidskoj ravnini poznato je da je produkt tri simetrije ponovo simetrija ako i samo ako se njihove osi sijeku u jednoj zajedničkoj točki. Također poznat je i neeuklidski analogon "teorema tri simetrije" (3RT), vidi npr. [4]. $\mathrm{U}$ ovom članku predstavljene su afine verzije tog teorema tako da se proučavaju tri mimosmjerne simetrije kojima se osi sijeku u jednoj točki. Pokazat će se da je važno, u svim verzijama 3RT-a, da se tri para (os, smjer simetrije) danih (mimosmjernih)simetrija mogu proučavati kao involutivni projektivitet. Za euklidski i neeuklidski slučaj ovo svojstvo je automatski ispunjeno.

Sa stajališta projektivne geometrije (mimosmjerna) simetrija je harmonička homologija. U afinoj geometriji simetrija je indirektna involutivna transformacija, dok u projektivnoj geometriji nema smisla govoriti o "direktnoj" i "indirektnoj" transformaciji. Harmonička homologija dopušta interpretaciju i kao osnu simetriju i kao centralnu simetriju. Ipak, može se proučavati produkt triju harmoničkih homologija koji je ponovno harmonička homologija. Nekim posebnim međusobnim položajima centara i osi danih homologija može se dobiti elacija ili čak identitet.

Posljedica danih rezultata su daljnje generalizacije 3RT-a, npr. u ravninama s Minkowski metrikom, afinim ili projektivnim 3-dimenzionalnim prostorima ili u geometrijama kružnice.

Ključne riječi: teorem tri simetrije, osna simetrija, harmonička homologija, involutivni projektivitet

tion of such a sub-geometry is the (finite) product of reflections, and so the question arises, under which conditions is such a product again a reflection. For the product of three reflections this leads to the conditions described as "Three Reflections Theorem" (3RT) in a Euclidean or (non-Euclidean) Cayley-Klein planes, see e.g. [4], [5]. 
In the following chapter we will state the known facts about the 3RT, providing the tools for further generalisations. Chapter 3 shortly refers to the non-Euclidean case and to circle geometries, while the following chapters present seemingly new generalisations of the 3RT in affine and projective planes and spaces.

\section{Basic facts about the 3RT}

We start with the Euclidean plane $\Pi$ as place of action. A (line) reflection $\sigma: \Pi \rightarrow \Pi$ is an indirect involutoric congruence transformation of $\Pi$; the set of fixed points is a line, the "axis" $a$ of $\sigma$, the set of fixed lines consists of $a$ and a pencil of parallels orthogonal to $a$. For an arbitrary congruence transformation yield the following two theorems:

Theorem 1 Each direct congruence transformation is either the identity 1 , a translation $\tau$, or a rotation $\rho$. Each indirect congruence transformation is either a slide reflection $\lambda$, or a (line) reflection $\sigma$.

Theorem 2 Each direct resp. indirect congruence transformation is the product of maximal 2 resp. 3 (line) reflections.

Special cases of direct congruence transformations are (1) the identity $l$, and (2) the rotation $\rho$ having rotation angle $\pi$. Such a rotation is called a "half turn" and it is a point reflection at the same time with a pencil of fixed lines through the (single) fixed centre of the half turn $\rho$.

Remark 1 Given two reflections $\sigma_{1}, \sigma_{2}$ with axes $a_{1}, a_{2}$ forming an angle $\measuredangle a_{1} a_{2}=: \alpha$, then the product $\sigma_{1} \sigma_{2}=\rho$ is a rotation with rotation angle $\delta=2 \alpha$ and exactly one fixed point. Reversely, the reflections $\sigma_{1}, \sigma_{2}$ to a given rotation $\rho$ are not uniquely determined. From this property it can be deduced that the product $\sigma_{1} \sigma_{2}=\rho$ is a halfturn $\Leftrightarrow \measuredangle a_{1} a_{2}=: \frac{\pi}{2}$.

For indirect congruence transformations reflections are the only special cases. Each slide reflection $\lambda$ is product of three reflections $\sigma_{i}$, which can be chosen such that two have parallel axes $a_{1}, a_{2}$ and the third $a_{3}$ is orthogonal to the former two and acting as the "slide axis". Thus the question arises about the conditions for the $a_{i}$ leading to a product reflection $\sigma_{1} \sigma_{2} \sigma_{3}$ instead of just a general slide reflection. The results are well-known:

Theorem 3 (the classical 3RT) $\sigma_{1} \sigma_{2} \sigma_{3}=: \sigma_{4}$ is a reflection $\Longleftrightarrow a_{1}\left\|a_{2}\right\| a_{3}$, or $a_{1} \cap a_{2} \cap a_{3}=\{A\}$.. common point.

As a key idea for a proof, which will be used also further on, we assume $\sigma_{1} \sigma_{2} \sigma_{3}=: \sigma_{4}$ to be a reflection. $\Rightarrow$ $\sigma_{1} \sigma_{2} \sigma_{3} \sigma_{4}=\sigma_{4} \sigma_{4}=i d \Rightarrow\left(\sigma_{1} \sigma_{2}\right)\left(\sigma_{3} \sigma_{4}\right)=i d \Rightarrow\left(\sigma_{1} \sigma_{2}\right)$ and $\left(\sigma_{3} \sigma_{4}\right)$ are inverse rotations or inverse translations. In other words, $\sigma_{3} \sigma_{4}$ must be just another description for $\sigma_{2} \sigma_{1}$. As a consequence, the angles $\measuredangle a_{1} a_{2}$ and $\measuredangle a_{3} a_{4}$ of their axes $\alpha_{i}$ must be equal and the $\cap a_{i}=\{A\}$ resp. $\operatorname{dist}\left(a_{1}, a_{2}\right)=\operatorname{dist}\left(a_{3}, a_{4}\right)$ for parallel $a_{i}$.

\section{Non-Euclidean and circle-geometric ver- sions of the 3RT}

a) As this chapter still concerns known facts, we restrict ourselves to presenting some examples starting with the hyperbolic plane as a place of action. We use the Kleinmodel, i.e. the full projective plane endowed with an absolute hyperbolic polarity according to e.g. N. Wildberger [7]. A hyperbolic reflection $\sigma$ is a harmonic homology of the (regular) absolute conic $\omega$. This means that the axis $a$ of $\sigma$ is not tangent to $\omega$. Here again the product $\sigma_{1} \sigma_{2} \sigma_{3}=: \sigma_{4}$ is a reflection $\Leftrightarrow a_{1} \cap a_{2} \cap a_{3}=\{A\}$, see Figure 1. We omit the discussion of special cases.

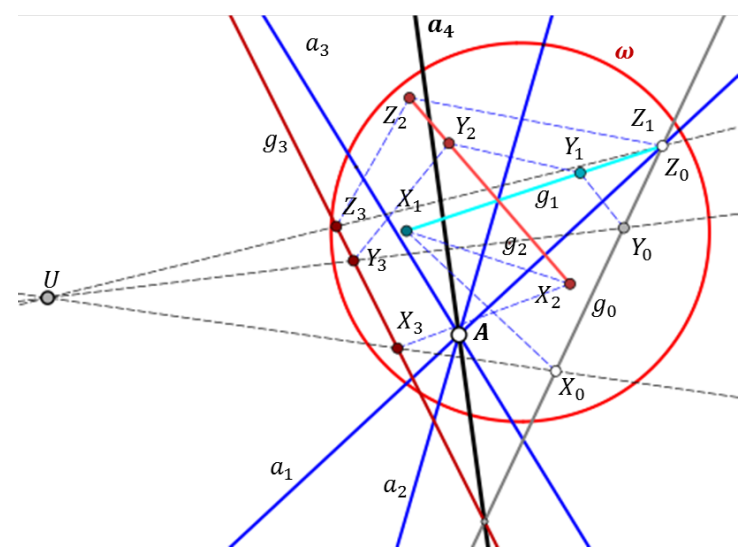

Figure 1: Visualisation of the hyperbolic version of the $3 R T$.

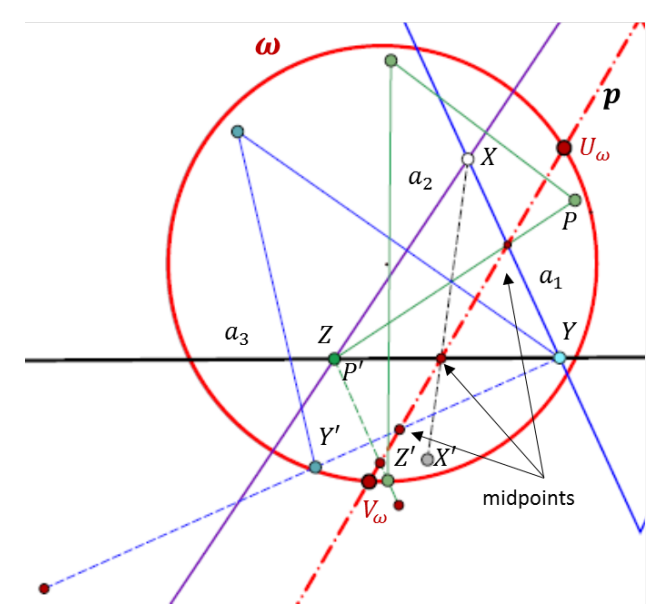

Figure 2: The product of three hyperbolic reflections induces a projectivity in the absolute conic $\omega$. 
The general case then means that the three given axes $a_{i}$ form a trilateral. Figure 2 shows the case of an inner trilateral $\left(a_{1} a_{2} a_{3}\right)$ of $\omega$ : The product $\sigma_{1} \sigma_{2} \sigma_{3}$ induces a (hyperbolic) projectivity $\pi$ at $\omega$ with projectivity axis $p$ and fixed points $U_{\omega}, V_{\omega}$.

The construction of the projectivity axis $p$ turns out to be an analogue to the construction of the Euclidean slidereflection axis (Figure 3): $p$ passes through midpoints of segments formed by pairs of homologuos points $X, X^{\prime}=$ $X^{\sigma_{1} \sigma_{2} \sigma_{3}}$

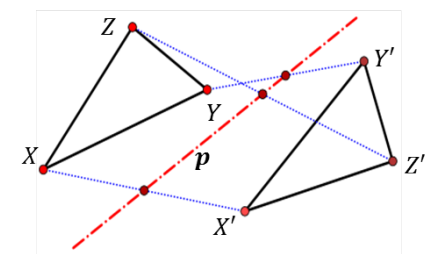

Figure 3: Construction of the slide-reflection axis $p$ in the Euclidean plane.

b) As an example for a circle geometry place of action we will use the Euclidean Möbius case. "Reflection" $\sigma$ now represents an inversion at a Möbius circle $a$.

A "Möbius three reflections theorem" then reads as follows:

Theorem 4 (Möbius 3RT) $\sigma_{1} \sigma_{2} \sigma_{3}=: \sigma_{4}$ is an inversion $\Longleftrightarrow a_{1}, a_{2}, a_{3} \in$ common pencil of Möbius circles.

As before, $\sigma_{1} \sigma_{2} \sigma_{3} \sigma_{4}=\sigma_{4} \sigma_{4}=i d \Leftrightarrow \sigma_{3} \sigma_{4}=\sigma_{2} \sigma_{1}$, inverse to $\sigma_{1} \sigma_{2}$, which is a "Möbius rotation". And, as in the Euclidean case, the rotation angle is twice the (real or imaginary) intersection angle between the two fixed circles $a_{1}, a_{2}$. Figure 4, 5 and 6 visualise such Möbius rotations generated by two inversions.

Recently E. Molnar [4] treated also non-Euclidean circle geometries according to F. Bachmann's point of view, namely generating a geometry via reflections, c.f. [2].

Projective geometric generalisations of the concept inversion seem to trace back to Thomas Archer Hirst (1830 1892 ), even though there are hardly any references to be found:

Definition 1 A "Hirst inversion" 1 is an involutoric mapping of the n-dimensional projective space $P^{n}$ where a centre point $O$ and a polarity $\pi: P^{n} \rightarrow P^{n}$ is given, such that (1) $O, X, X^{\mathrm{l}}$ are collinear, and (2) $X, X^{\mathrm{l}}$ are conjugate points in $\pi,(X \neq O)$.

Remark 2 This version of defining a Hirst inversion was presented by H. Brauner (1928 - 1990) in his unfortunately unpublished "Lectures on Geometry". How to arrange three Hirst inversions such that their product again is a Hirst inversion seems to be an open problem.

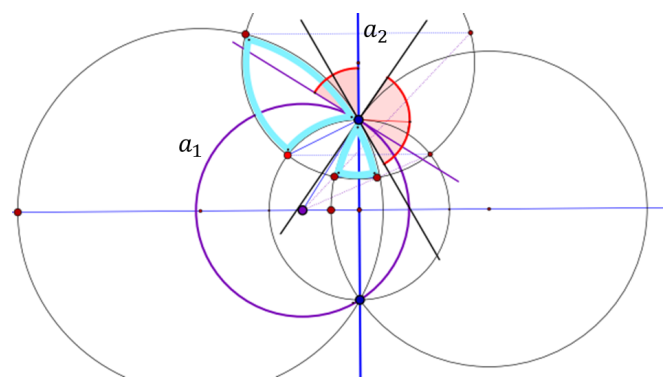

Figure 4: $a_{1}, a_{2}$ span an elliptic pencil of Möbius circles. The rotation angle of the product $\sigma_{1} \sigma_{2}$ is twice the angle $\measuredangle a_{1} a_{2}$.

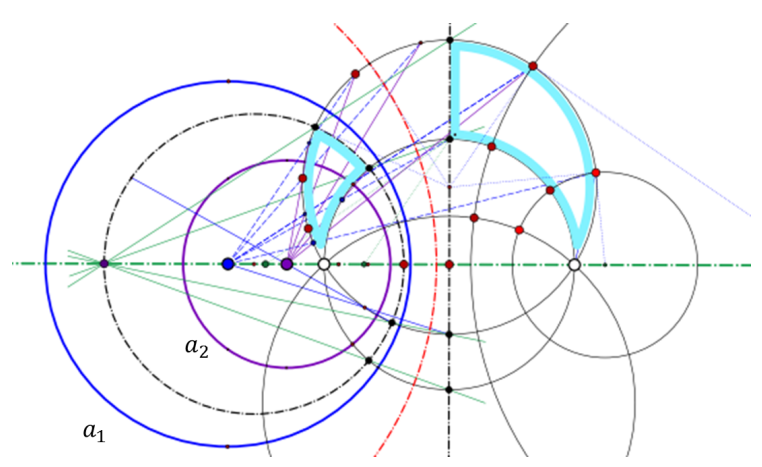

Figure 5: $a_{1}, a_{2}$ span a hyperbolic pencil of Möbius circles. The rotation angle of the product $\sigma_{1} \sigma_{2}$ is imaginary, but still twice the angle $\measuredangle a_{1} a_{2}$.

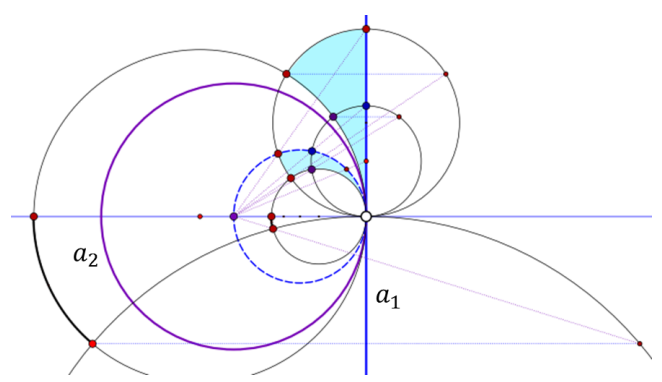

Figure 6: The limit rotation case: $a_{1}, a_{2}$ span a parabolic pencil of Möbius circles.

\section{The affine 3RT}

Place of action is now an affine plane. Let its coordinate field be a commutative field $\mathcal{F}$ with $\operatorname{char} \mathcal{F} \neq 2,3$. As we deal with (general) reflections, we consider affine transformations $\eta$ of $e S L(2, \mathcal{F})$, i.e. they have coordinate representations by matrices with det $= \pm 1$.

Remark 3 A (skew) reflection $\sigma$ is an involutoric perspective affine transformation with axis $a$ and a pencil of parallel fixed lines $\{b \ldots\}$ not containing a. Obviously, "skew" without a concept of orthogonality does not make sense. Again, we distinguish direct-and indirect-affine transformations $\eta \in e S L(2, \mathcal{F})$. 
We summarize the well-known facts

Theorem 5 Each direct transformation $\eta \in e S L(2, \mathcal{F})$ is either (1) the identity, (2) a translation, (3) a shear (transvection), or (4) an affine rotation. Each indirect transformation $\eta \in e S L(2, \mathcal{F})$ is either (5) a shearreflection, or (6) a (line) reflection.

Theorem 6 Each $\eta \in e S L(2, \mathcal{F})$ is the product of maximal 3 (line) reflections. Each coordinate representation matrix of $\eta$ can therefore be factorised by matrices of three reflections $\sigma_{1}, \sigma_{2}, \sigma_{3}$.

Figure 7 shows the constructions of reflection axes $a_{i}$ and reflection-directions $b_{i}$ to given direct-affine resp. indirectaffine triangles $T, T^{\prime}$ having equal areas, while Figure 8 visualises the construction of a reflection $\sigma_{1}$ and a shear $\delta$ to two indirect-affine triangles.

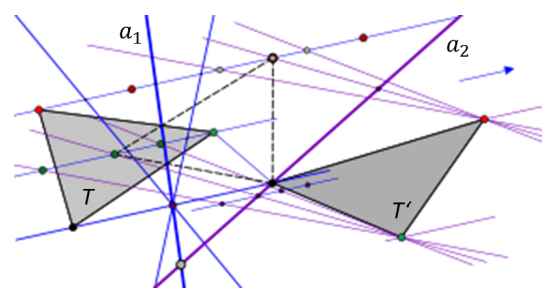

Figure 7: Construction of two reflections $\sigma_{1}, \sigma_{2}$ to directaffine triangles $T, T^{\prime}$ such that $\sigma_{1} \sigma_{2}: T \rightarrow T^{\prime}$.

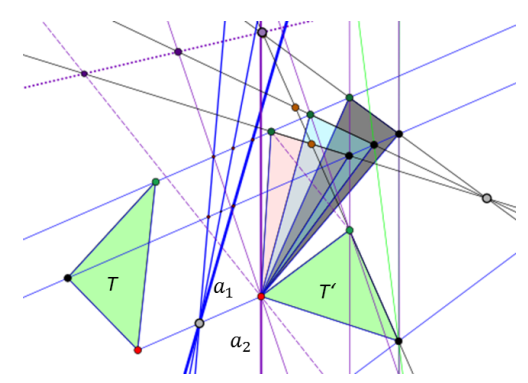

Figure 8: Construction of a reflection $\sigma$ and a shear $\delta$ to indirect-affine triangles $T, T^{\prime}$ such that $\sigma \delta$ : $T \rightarrow T^{\prime}$.

In the first, the direct case, one should find the second fixed point of a projectivity with known first fixed point. In the indirect case variation of $\sigma$ such that $\delta$ becomes a shear leads to a perspectivity. In both cases the construction deals only with linear graphic operations.

Now we ask, when the product of three affine reflections $\sigma_{1}, \sigma_{2}, \sigma_{3}$, is a reflection $\sigma_{1} \sigma_{2} \sigma_{3}=: \sigma_{4}$, too. According the key idea $\sigma_{1} \sigma_{2} \sigma_{3} \sigma_{4}=\sigma_{4} \sigma_{4}=i d$ the first two factors, $\sigma_{1} \sigma_{2}$, and the last two ones, $\sigma_{3} \sigma_{4}$, must define inverse affine transformations.
General case: $\sigma_{1}, \sigma_{2}$ such that there exists exactly one proper fixed point $A$, that means, $\sigma_{1} \sigma_{2}$ is an "affine rotation". (Note that for a reflection $\sigma(a, b)$ the axis $a$ and direction of the reflection $b$ are different.) Furthermore, we consider three reflections $\sigma_{i}, i=1,2,3$, with axes $a_{i}$ and reflection-directions $b_{i}$ such that all six directions are different.

As a necessary condition for $\sigma_{1} \sigma_{2} \sigma_{3}=: \sigma_{4}$ being a reflection, too, we get that $a_{3}$ must pass through $A$. The pairs $\left(a_{1}, b_{1}\right),\left(a_{2}, b_{2}\right)$ define an involutoric projectivity $\mu$ and $\left(a_{3}, b_{3}\right),\left(a_{4}, b_{4}\right)$ must define the same $\mu$. This leads to the sufficient condition that all three pairs $\left(a_{1}, b_{1}\right),\left(a_{2}, b_{2}\right)$, $\left(a_{3}, b_{3}\right)$ are pairs of an involutoric projectivity $\mu$. This gives

Theorem 7 Necessary and sufficient condition for an "affine 3RT" (in the general case) is that the given three reflections $\sigma_{1}, \sigma_{2}, \sigma_{3}$ have axes $a_{i}$ and reflection-directions $b_{i}$ fulfilling the conditions $a_{1} \cap a_{2} \cap a_{3}=\{A\} \wedge\left(a_{1}, b_{1}\right)$, $\left(a_{2}, b_{2}\right),\left(a_{3}, b_{3}\right)$ are pairs of an involution $\mu$.

In planar Cayley-Klein geometries the sufficient condition "involution" $\mu$ is automatically fulfilled and therefore there is no need to mention it.

Special case: $\sigma_{1}, \sigma_{2}$ have exactly one proper fixed point $A$ and $a_{1} \in\left\{b_{2}\right\} \wedge a_{2} \in\left\{b_{1}\right\}$. That means, $\sigma_{1} \sigma_{2}$ is a point reflection and the pairs $\left(a_{1}, b_{1}\right),\left(a_{2}, b_{2}\right)$ are identical and do not define an involution $\mu$. In this case we could start with $\sigma_{4} \sigma_{1} \sigma_{2} \sigma_{3}=\sigma_{4} \sigma_{4}=i d$ and have $\sigma_{2} \sigma_{3}$ as a proper affine rotation defining an involution $\mu$. Moreover, $\sigma_{3} \sigma_{4}$ is the inverse point reflection to $\sigma_{1} \sigma_{2}$.

We omit the discussion of further cases.

\section{A 3RT in normed planes}

Real affine planes can also be endowed with a norm based on a convex, centrally symmetric "unit circle" $c$. Such a plane is called the Minkowski plane $\Pi_{c}$ and there exist several possibilities to define an orthogonality in $\Pi_{c}$. Most common is the Birkhoff's left-orthogonality, which is nonsymmetric. For Minkowski geometry see e.g. [1], [3], [6] and [8].

Definition 2 A Minkowski (line) reflection is an involutoric affine reflection, whereby the reflection-direction $b$ is left-orthogonal to the axis a.

Note that a Minkowski reflection $\sigma$ is, in general, not norm preserving, as the unit circle $c$ and its image $c^{\sigma}$ are, in general, not translatoric congruent. Nevertheless, one could study products of three Minkowski reflections:

Theorem 8 (Minkowski-3RT) The product of three Minkowski reflections $\sigma_{1}, \sigma_{2}, \sigma_{3}$ is a Minkowski reflection $\sigma_{4} \Longleftrightarrow \sigma_{1}, \sigma_{2}, \sigma_{3}$ fulfill the affine $3 R T$ conditions and $\sigma_{1} \sigma_{2} \sigma_{3}=: \sigma_{4}$ fulfills the left-orthogonality condition. 
For a general unit circle $c$ and two given Minkowski reflections $\sigma_{1}, \sigma_{2}$ there will, in general, not exist a $\sigma_{3}$ such that $\sigma_{1}, \sigma_{2}, \sigma_{3}$ fulfill the affine 3RT conditions. For an arbitrary unit circle $c$ there exists at least one pair of "conjugat" diameters such that left-orthogonality is symmetric, see [6]. In case $c$ has two such pairs, the first one can act as $a_{1}, a_{2}$ and the second one as $a_{3}, a_{4}$, similar to the special case described in the former chapter. Figure 9 shows such a case with a smooth unit circle $c$.

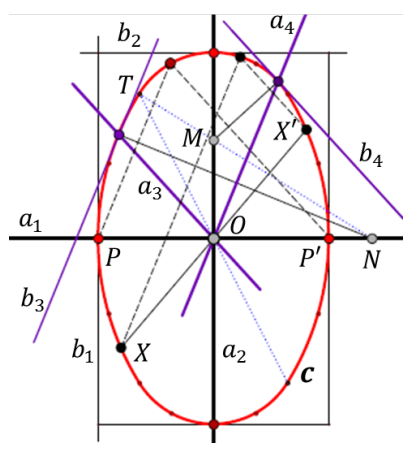

Figure 9: The Minkowski unit circle c consisting of circular bi-arcs possesses a discrete number of conjugate diameters, which can be used as axes for a valid Minkowski-3RT.

Special cases can occur for affine regular $2 n$-gons as unit circles $c$, see Figure 10.

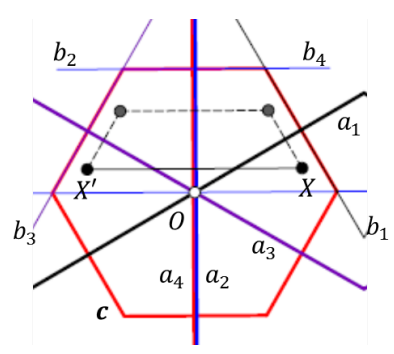

Figure 10: For an affine-regular hexagon as Minkowski unit circle $c$ and Minkowski reflections $\sigma_{1}, \sigma_{2}$, $\sigma_{3}$ with symmetry axes of $c$ as axes $a_{i}$ the product reflection $\sigma_{4}$ coincide with $\sigma_{2}$.

To conclude we can find cases of normed planes, where a Minkowski-3RT is valid for specially chosen Minkowski reflections $\sigma_{1}, \sigma_{2}, \sigma_{3}$.

\section{The 3RT in affine 3-spaces}

Place of action is now a three-dimensional affine space $\Pi^{3}$ with a commutative coordinate field $\mathcal{F}$ and $\operatorname{char} \mathcal{F} \neq 2,3$. As we deal with (plane) reflections, we consider affine transformations $\eta \in e S L(3, \mathcal{F})$, i.e. they have coordinate representations by matrices with det $= \pm 1$.
If $\mathcal{F} \cong \mathbb{R}$ and $\Pi^{3}$ is a Euclidean 3-space, then from Chapters 3 and 4 follows immediately

Theorem 9 The necessary and sufficient condition for the $3 R T$ in a Euclidean 3-space is that $\sigma_{1}, \sigma_{2}, \sigma_{3}$ have axis planes $a_{1}, a_{2}, a_{3}$ which span a pencil of planes.

For an affine reflection $\sigma$ in a general affine 3 -space $\Pi^{3}$ the reflection-direction $b$ is not parallel to the fixed plane $a$. As a consequence of Theorem 5 the following theorem, which describes the general case, is obvious:

Theorem 10 Necessary and sufficient conditions for an affine 3RT in an affine 3-space $\Pi^{3}$ are: The three reflections $\sigma_{1}, \sigma_{2}, \sigma_{3}$ have axes $a_{i}$ and reflection-directions $b_{i}$ fulfilling

(1) $a_{1} \cap a_{2} \cap a_{3}=:$ a . . axis of a pencil of planes,

(2) $b_{1}, b_{2}, b_{3}$ are parallel to a plane $\gamma \wedge \gamma \nmid a$,

(3) $\gamma \cap a_{i}=: c_{i},\left(c_{1}, b_{1}\right),\left(c_{2}, b_{2}\right),\left(c_{3}, b_{3}\right)$ are pairs of an involution $\mu$.

For some special cases of the given reflections $\sigma_{1}, \sigma_{2}, \sigma_{3}$ the Theorems 7 and 8 have to be modified. We omit a complete discussion of such cases.

One could also extend the original question, when the product of three reflections $\sigma_{1}, \sigma_{2}, \sigma_{3}$ is a reflection to a product being a special affine transformation. For example, in the Euclidean 3-space, the reflections at pairwise orthogonal planes $a_{i}$ lead to a point reflection. We will meet such cases in Chapter 8 .

The general direct-affine transformation $\eta \in e S L(3, \mathcal{F})$ is the product of 4 reflections, while indirect-affine transformations can be factorised by 3 reflections. The constructive treatment of these factorisations is a problem of its own.

\section{Projective geometric interpretation of the planar 3RT: the 3HHT}

Place of action is a projective plane $\Pi$. Let its coordinate field be a commutative field $\mathcal{F}$, char $\mathcal{F} \neq 2,3$, for example $\mathcal{F} \cong \mathbb{R}$. "Reflections" $\sigma_{i}$ now represents harmonic homologies of $P G L(\Pi, \mathcal{F})$ with axis $a_{i}$ and centre $B_{i}$. Note that the concepts "direct" or "indirect" do not make sense anymore. Harmonic homologies are point reflections and line reflections at the same time.

Applying the key idea that from the assumption $\sigma_{1} \sigma_{2} \sigma_{3}=$ : $\sigma_{4}$ is a harmonic homology it follows $\sigma_{1} \sigma_{2} \sigma_{3} \sigma_{4}=\sigma_{4} \sigma_{4}=$ $i d$. This means that $\sigma_{1} \sigma_{2}$ and $\sigma_{3} \sigma_{4}$ must be inverse collineations, and therefore we will, at first, study the product of two harmonic homologies:

We start with the general case of $\sigma_{1}\left(a_{1}, B_{1}\right), \sigma_{2}\left(a_{2}, B_{2}\right)$ represented by Figure 11. The line $s$ connecting the centres $B_{i}$ of $\sigma_{i}$ is a fixed line of the product collineation $\sigma_{1} \sigma_{2}=: \kappa$, 
the intersection point $S$ of the axes $a_{i}$ is a fixed point of $\kappa$. The other (real or imaginary) fixed points $R, T$ are fixed points of the involution $\mu$ defined by the two pairs $\left(B_{1}, A_{1}:=s \cap a_{1}\right)$ and $\left(B_{2}, A_{2}:=s \cap a_{2}\right)$. By mapping consecutively a point $X$ to $X^{\prime}$ and $Y:=X^{\prime}$ to $Y^{\prime} \ldots$ we obtain a series of points of a conic. This justifies to call $\kappa$ a "projective rotation".

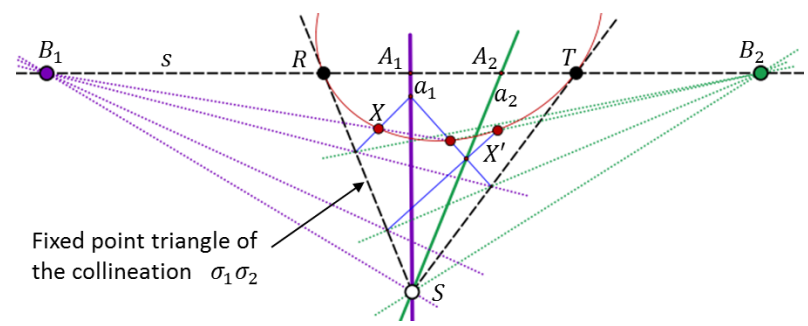

Figure 11: Product collineation of two harmonic homologies

The mentioned general case describes the classical situation for the Euclidean and affine 3RT, if $s$ is interpreted as the ideal line of the place of action. Therefore it follows

Theorem 11 (3HHT) Let two harmonic homologies $\sigma_{1}$, $\sigma_{2}$ be in general position. The necessary and sufficient conditions that the product with a third harmonic homology $\sigma_{3}$ is a harmonic homology $\sigma_{4}$ again, is

(1) $B_{3} \in s:=B_{1} B_{2}$ and $\cap a_{i}=\{S\}, i=1,2,3$, where $B_{i}$ are centers and $a_{i}$ axes of $\sigma_{i}$,

(2) $\left(A_{1}, B_{1}\right),\left(A_{2}, B_{2}\right),\left(A_{3}, B_{3}\right)$ are pairs of an involution $\mu$ in $s,\left(A_{i}:=a_{i} \cap s\right)$.

Among the many special cases of mutual positions of $\left\{\left(B_{1}, a_{1}\right),\left(B_{2}, a_{2}\right),\left(B_{3}, a_{3}\right)\right\}$ we mention the following:

a) $\sigma_{1}, \sigma_{2}$ with $B_{1} \in a_{2} \wedge B_{2} \in a_{1}$.

In this case, the product $\sigma_{1} \sigma_{2}=\sigma$ is already a harmonic homology with centre $S=a_{1} \cap a_{2}$ and axis $s=B_{1} B_{2}$. If we choose $\sigma_{3}=\sigma$, then the product $\sigma_{1} \sigma_{2} \sigma_{3}=\sigma_{4}=\imath$ is the identity. For $B_{3} \in B_{1} B_{2}$ and $a_{3} \ni a_{1} \cap a_{2}$ condition (1) is the only condition for $\sigma_{1} \sigma_{2} \sigma_{3}=\sigma_{4}$ being a harmonic homology.

b) $\sigma_{1}, \sigma_{2}$ with $a_{1}=a_{2}$ and $B_{1}, B_{2}$ arbitrary.

In this case $a_{3}$ must coincide with $a_{1}=a_{2}$ and we have a common fixed axis, while $B_{3}$ can be chosen arbitrarily, see fig. 12. This is the classical situation for the product of three point-reflections in the $\mathrm{Eu}-$ clidean plane and also in the affine plane. Obviously, the following extension to higher dimensions holds too:
Theorem 12 Three harmonic homologies $\sigma_{1}, \sigma_{2}, \sigma_{3}$ in a projective $n$-space with coinciding hyperplanes $a_{i}$ in a projective $n$-space have a harmonic homology $\sigma_{4}=\sigma_{1} \sigma_{2} \sigma_{3}$ as their product.

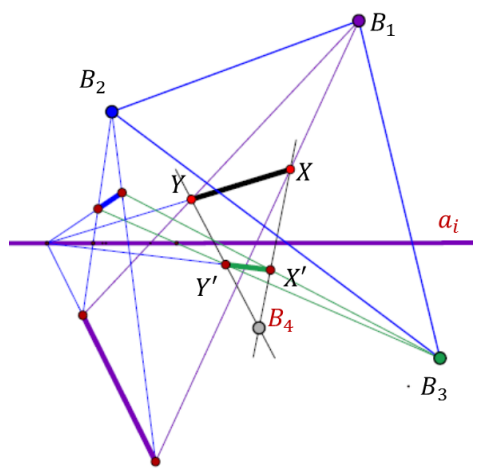

Figure 12: The product of three harmonic homologies with coinciding axes is a harmonic homology.

The dual situation, namely coinciding centres $B_{i}$ and arbitrarily chosen axes $a_{i}$ leads to the dual version of Theorem 10.

\section{Products of harmonic axial collineations and axial reflections}

Extending the Three Reflections Theorem to spaces of higher dimensions, as e.g. formulated as Theorem 10, induces an additional idea: Let us generalise $\sigma_{i}$ to harmonic axial collineations in $n$-dimensional projective spaces $\Pi^{n}$. We assume the fixed spaces $a_{i}, b_{i}$ of $\sigma_{i}$ to be skew and complementary subspaces of $\Pi^{n}$, i.e. $\operatorname{dim} a_{i}=n-d_{i}-1$, $\operatorname{dim} b_{i}=d_{i}$. We even might combine axial collineations $\sigma_{i}, \sigma_{j}$ with $\operatorname{dim} b_{i} \neq \operatorname{dim} b_{j}$. But already for dimension $n=4$ we would not get an even remotely comprehensible set of cases and subcases to deal with. Therefore, we restrict the place of action to a projective 3 -space $\Pi^{3}$ and its coordinate field to $\mathbb{R} \subset \mathbb{C}$. Furthermore, we will only treat the case $d_{i}=d_{j}=1$, i.e. the pair of axes $a, b$ of a harmonic axial collineation $\sigma$ that are skew lines. As such a collineation is involutoric it is also called an "axial involution".

As in the former chapters, we pose the question, under which conditions the product $\sigma_{1} \sigma_{2} \sigma_{3}=: \sigma_{4}$ turns out to be an axial involution. Assuming $\sigma_{1} \sigma_{2} \sigma_{3}=: \sigma_{4}$ to be a reflection it leads to inverse collineations $\sigma_{1} \sigma_{2}$ and $\sigma_{3} \sigma_{4}$. With this in mind, we first have to study the product $\kappa$ of two axial involutions $\sigma_{1}, \sigma_{2}$.

We start with the general case $\sigma_{1}\left(a_{1}, b_{1}\right), \sigma_{2}\left(a_{2}, b_{2}\right)$ with $\left\{a_{1}, b_{1}, a_{2}, b_{2}\right\}$ spanning a $2 \mathrm{D}$-set of lines, such a set is called a line congruence), see Figure 13: 


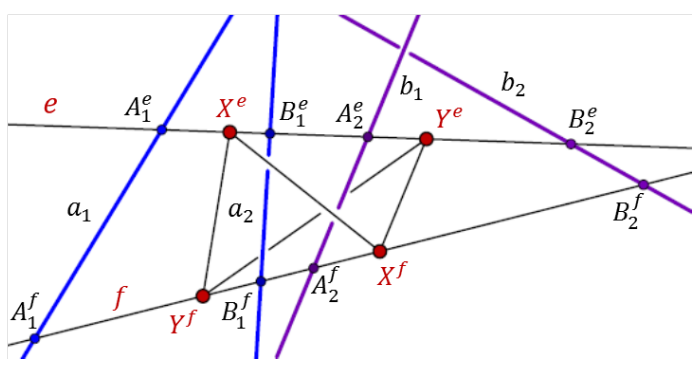

Figure 13: Sketch visualizing two harmonic axial collineations and the fixed point tetrahedron of their product collineation.

There are two (real, imaginary or coinciding) lines $e, f$ meeting all four lines $a_{1}, b_{1}, a_{2}, b_{2}$. Let us consider the case, when $e, f$ are real and distinct. The construction of such lines is known as the H. E. Timerding problem. Thereby, one intersects the quadric $\Phi$ defined by three lines $a_{1}, b_{1}, a_{2}$ with the additional line $b_{2}$. The generators $\Phi$ through the intersection points and not belonging to the same regulus of $\Phi$, as $a_{1}, b_{1}, a_{2}$, are the lines $e, f$ intersecting all four given lines.

The lines $e, f$ are fixed lines of $\kappa$; we label their intersection points with the axes $a_{i}, b_{i}$ by $A_{i}^{e}, A_{i}^{f}$ and $B_{i}^{e}, B_{i}^{f}$. The pairs $\left(A_{1}^{e}, B_{1}^{e}\right)$ and $\left(A_{2}^{e}, B_{2}^{e}\right)$ define an involutoric projectivity $\mu^{e}$ in, the fixed points (over $\mathbb{C}$ ) $X^{e}, Y^{e}$ which are fixed points of $\kappa$. The analogue procedure for the line $f$ results in the remaining fixed points (over $\mathbb{C}$ ) $X^{f}, Y^{f}$ of $\kappa$. By mapping an admissible point $P \mapsto P^{\sigma_{1} \sigma_{2}}$ we finally receive a well-defined collineation $\kappa$.

The conditions to be fulfilled by a third harmonic axial collineation $\sigma_{3}\left(a_{3}, b_{3}\right)$ such that $\sigma_{1} \sigma_{2} \sigma_{3}=: \sigma_{4}$ is again an axial collineation are then obvious:

Theorem 13 (axial 3HHT) The product $\sigma_{1} \sigma_{2} \sigma_{3}=$ : $\sigma_{4}$ of three generally positioned harmonic axial collineations $\sigma_{1}, \sigma_{2}, \sigma_{3}$ is again a harmonic axial collineation, if and only if their axes $a_{i}, b_{i}$ belong to a line congruence (i.e. they span a $2 D$-set of lines) with axes $e, f$ (over $\mathbb{C}$ ) and the intersection pairs $\left(A_{i}^{e}, B_{i}^{e}\right)$ and $\left(A_{i}^{f}, B_{i}^{f}\right)$ with these axes corresponds to involutions $\mu^{e}, \mu^{f}$ on e and $f$.

Note that the product collineation $\kappa$ of two axial involutions $\sigma_{1}, \sigma_{2}$ generates a subgroup $\left\{\kappa^{n}, n \in \mathbb{Z}\right\} \subset$ $P G L\left(\Pi^{3}, \mathbb{R} \subset \mathbb{C}\right)$, namely the one keeping the quadrics of a pencil of quadrics fixed. This is an analogue to the planar case of projective rotations, as we mentioned in Chapter 7. Among the many cases of mutual positions of $\sigma_{1}\left(a_{1}, b_{1}\right)$, $\sigma_{2}\left(a_{2}, b_{2}\right)$ the one with $\left\{a_{1}, b_{1}, a_{2}, b_{2}\right\}$ spanning a $1 \mathrm{D}$-set of lines, (namely a regulus on a quadric $\Phi$ ) shall at least be mentioned. In this case the set of fixed lines is the complementary regulus and the product $\sigma_{1} \sigma_{2}=: \kappa$ already is an axial collineation. A third harmonic axial collineation $\sigma_{3}$ then must have axes belonging to the same regulus as $a_{1}$, $b_{1}, a_{2}, b_{2}$ such that $\sigma_{1} \sigma_{2} \sigma_{3}=: \sigma_{4}$ is again a harmonic axial collineation.

Another very special case of mutual positions of $\sigma_{1}\left(a_{1}, b_{1}\right), \sigma_{2}\left(a_{2}, b_{2}\right)$ would be that lines $a_{1}, b_{1}, a_{2}, b_{2}$ form a skew quadrilateral. Interpreting the plane $b_{1} \vee b_{2}$ as the ideal plane of the Euclidean 3-space and $a_{1}, a_{2}$ as (intersecting) orthogonal lines, we might speak of $\sigma_{1}, \sigma_{2}$ as axial reflections. The product $\sigma_{1} \sigma_{2}=: \kappa$ is then an axial reflection at an axis orthogonal to $a_{1} \vee a_{2}$ and passing through $a_{1} \cap a_{2}$. Choosing $\kappa$ as the third axial reflection gives then the identity as the product $\sigma_{1} \sigma_{2} \sigma_{3}$.

Finally, we consider the case of axial reflections $\sigma_{1}, \sigma_{2}, \sigma_{3}$ whereby $a_{i}$ are skew edges of a cube. In this case the product $\sigma_{1} \sigma_{2} \sigma_{3}$ turns out to be a translation $\tau$, see Figure 14 .

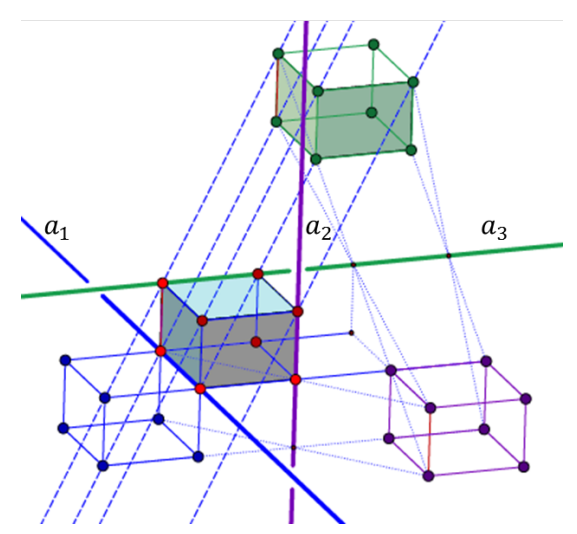

Figure 14: The product of three axial reflections at pairwise orthogonal axes is a translation.

\section{Conclusion}

The Three Reflections Theorem belongs to basic and wellknown geometric facts. But as is shown in the chapters above, there are still surprising results to gain, when we generalise the place of action. Leading idea is Bachmann's point of view of generating a geometry via reflections, even when it in most cases defines only an important sub-geometry of "classical" geometries. There is a unifying treatment for all mentioned generalisations: we study products of two reflections first and choose the third such that it, together with a fourth, gives the inverse product. This makes it possible to omit explicit calculations.

\section{Acknowledgement}

The author thanks Prof. E. Jurkin and the reviewer(s) for valuable help and hints. 


\section{References}

[1] J. Alonso, C. Benitez, Orthogonality in normed linear spaces. Part 1, Extracta Math. Scripta Math. 3(1) (1988), 1-15, and Part 2, Extracta Math. 4(3) (1989), 121-131.

[2] F. Bachmann, Aufbau der Geometrie aus dem Spiegelungsbegriff, 2nd ed., Springer Verlag Heidelberg, 1973.

[3] G. BIRKHOFF, Orthogonality in linear metric spaces, Duke Math. J. 1 (1935), 169-172.

[4] E. Molnar, On non-Euclidean circle (sphere) geometry by reflections, Mongeometrija 2018, Novi Sad, Serbia. 2018.

[5] E. Molnar, Inversion auf der Idealebene der Bachmanschen metrischen Ebene, Acta Math. Acad. Sci. Hung. 37 (1981), 451-470.
[6] A.C. Thompson, Minkowski Geometry, Cambridge Univ. Press, 1996.

[7] N.J. WiLDBERGER, Universal Hyperbolic Geometry II, $K o G 14$ (2010), 3-24.

[8] I.M. YAGLOM, On the circular transformations of Möbius, Laguerre, and Lie, in: The Geometric Vein, The Coxeter Festschrift, Eds. C. Davis, B. Grünbaum, F.A. Sherk, 1981, 345-354.

\section{Gunter Weiss}

e-mail: weissgunter@gmx.at

University of Technology Vienna,

Karlsplatz 13, 1040 Vienna, Austria

University of Technology Dresden,

Helmholtzstrße 10, 01069 Dresden, Germany 